\title{
Notes on the roots of Steiner polynomials
}

\section{Martin Henk and María A. Hernández Cifre}

Dedicated to Jörg M. Wills on the occasion of his 70th birthday

\begin{abstract}
We study the location and the size of the roots of Steiner polynomials of convex bodies in the Minkowski relative geometry. Based on a problem of Teissier on the intersection numbers of Cartier divisors of compact algebraic varieties it was conjectured that these roots have certain geometric properties related to the in- and circumradius of the convex body. We show that the roots of 1-tangential bodies fulfill the conjecture, but we also present convex bodies violating each of the conjectured properties.
\end{abstract}

\section{Introduction}

Let $\mathcal{K}^{n}$ be the set of all convex bodies, i.e., compact convex sets, in the $n$ dimensional Euclidean space $\mathbb{R}^{n}$, and let $B_{n}$ be the $n$-dimensional unit ball. The subset of $\mathcal{K}^{n}$ consisting of all convex bodies with non-empty interior is denoted by $\mathcal{K}_{0}^{n}$. The volume of a set $M \subset \mathbb{R}^{n}$, i.e., its $n$-dimensional Lebesgue measure, is denoted by $\mathrm{V}(M)$. For two convex bodies $K, E \in \mathcal{K}^{n}$ and a non-negative real number $\rho$ the volume of $K+\rho E$ is expressed as a polynomial of degree $n$ in $\rho$ and it can be written as

$$
\mathrm{V}(K+\rho E)=\sum_{i=0}^{n}\left(\begin{array}{c}
n \\
i
\end{array}\right) \mathrm{W}_{i}(K ; E) \rho^{i} .
$$

This expression is called the Minkowski-Steiner formula or relative Steiner formula of $K$. The coefficients $\mathrm{W}_{i}(K ; E)$ are called the relative quermassintegrals of $K$, and they are just a special case of the more general defined

2000 Mathematics Subject Classification: Primary: 52A20, 52A39; Secondary: 30C15. Keywords: Steiner polynomial, Teissier's problem, tangential bodies, circumradius, inradius. 
mixed volumes for which we refer to $[13$, s. 5.1]. In particular, we have $\mathrm{W}_{0}(K ; E)=\mathrm{V}(K), \mathrm{W}_{n}(K ; E)=\mathrm{V}(E)$ and $\mathrm{W}_{i}(K ; E)=\mathrm{W}_{n-i}(E ; K)$.

If $E=B_{n},(1.1)$ becomes the classical Steiner formula [14], and $\mathrm{W}_{i}\left(K ; B_{n}\right)$, for short denoted by $\mathrm{W}_{i}(K)$, is the classical $i$-th quermassintegral of $K$. In this case, $n \mathrm{~W}_{1}(K)$ is the surface area of $K, \mathrm{~W}_{n}(K)=\mathrm{V}\left(B_{n}\right)=\kappa_{n}$ is the $n$-dimensional volume of $B_{n}$ and $\left(2 / \kappa_{n}\right) \mathrm{W}_{n-1}(K)$ is the mean width of $K[13$, p. 42].

The relative inradius $\mathrm{r}(K ; E)$ and relative circumradius $\mathrm{R}(K ; E)$ of $K$ with respect to $E$ are defined, respectively, by

$$
\begin{aligned}
\mathrm{r}(K ; E) & =\max \left\{r: \exists x \in \mathbb{R}^{n} \text { with } x+r E \subseteq K\right\}, \\
\mathrm{R}(K ; E) & =\min \left\{R: \exists x \in \mathbb{R}^{n} \text { with } K \subseteq x+R E\right\} .
\end{aligned}
$$

Notice that it always holds

$$
\mathrm{r}(K ; E) \mathrm{R}(E ; K)=1 .
$$

In the planar case the inradius, circumradius and the quermassintegrals are related by the well-known Bonnesen inequality

$$
\mathrm{W}_{1}(K ; E)^{2}-\mathrm{W}_{0}(K ; E) \mathrm{W}_{2}(K ; E) \geq \frac{\mathrm{W}_{2}(K ; E)^{2}}{4}(\mathrm{R}(K ; E)-\mathrm{r}(K ; E))^{2}
$$

Bonnesen [3] proved this result for $E=B_{2}$, the proof of the general case is due to Blaschke [2, pp. 33-36]. This inequality sharpens (in the plane) the Aleksandrov-Fenchel and the isoperimetric inequalities and there is no known generalization of it to higher dimensions. In fact (1.3) is an immediate consequence of the following stronger relations [2, pp. 33-36] (see also [6])

$$
\begin{array}{r}
\mathrm{W}_{0}(K ; E)+2 \mathrm{~W}_{1}(K ; E) \rho+\mathrm{W}_{2}(K ; E) \rho^{2} \leq 0 \\
\quad \text { if }-\mathrm{R}(K ; E) \leq \rho \leq-\mathrm{r}(K ; E) .
\end{array}
$$

The left hand side in this inequality is just the right hand side of the relative Steiner formula (1.1), and so (1.4) says that the so called (relative) Steiner polynomial

$$
f(K, E, s)=\sum_{i=0}^{n}\left(\begin{array}{c}
n \\
i
\end{array}\right) \mathrm{W}_{i}(K ; E) s^{i},
$$

regarded as a formal polynomial in a complex variable $s \in \mathbb{C}$, has in the case $n=2$ two real (negative) roots, one root less than or equal $-\mathrm{R}(K ; E)$ and the other root not less than $-\mathrm{r}(K ; E)$.

In [15] Teissier studied Bonnesen-Type inequalities in Algebraic Geometry, more precisely, intersection numbers of Cartier divisors of $n$-dimensional 
compact algebraic varieties. These intersection numbers "behave similarly" as the (relative) quermassintegrals and, in particular, in the case $n=2$ they satisfy an inequality similar to (1.4) with suitably defined in- and circumradius. Teissier raised the problem to find extensions of these two dimensional properties to higher dimensions (see also [10, p. 103]). In view of the properties derived from (1.4) in the planar case, in [11] and [12, p. 65] the following conjecture was posed which we formulate in terms of the relative Steiner polynomial. Furthermore, for a complex number $s$ we denote by $\operatorname{Re}(s)$ its real part.

Conjecture 1.1. Let $K, E \in \mathcal{K}^{n}$. If $\gamma_{i}, i=1, \ldots, n$, are the roots of the Steiner polynomial $f(K, E, s)$ with $\operatorname{Re}\left(\gamma_{1}\right) \leq \ldots \leq \operatorname{Re}\left(\gamma_{n}\right)$, then

$$
\operatorname{Re}\left(\gamma_{1}\right) \leq-\mathrm{R}(K ; E) \leq-\mathrm{r}(K ; E) \leq \operatorname{Re}\left(\gamma_{n}\right) \leq 0 .
$$

As mentioned before, (1.4) confirms the conjecture in dimension 2. A first systematic study of the roots of the classical Steiner polynomial in the 3dimensional case as well as their relations to the so called Blaschke diagram can be found in [7]. There it is also shown that the conjecture is correct for some special 3-dimensional convex bodies. But no further progress has been made on it; only some results have been obtained by Wills [16] for a closely related polynomial, the so called Wills functional.

In the following we will call the conjectured property that all real parts of the roots of $f(K, E, s)$ are non-positive the negativity property of the roots, and the conjectured bounds related to $\mathrm{r}(K ; E)$ and $\mathrm{R}(K ; E)$ will be referred to as the inradius and circumradius bound, respectively.

In Section 3 we study the above conjecture for the class of $p$-tangential bodies; for a definition see also Section 3. Among others we show that $n$-dimensional 1-tangential bodies, the so called cap-bodies, verify the conjecture.

Theorem 1.1. Let $K \in \mathcal{K}_{0}^{n}$ be a 1-tangential body of $E \in \mathcal{K}_{0}^{n}$. Then the roots of $f(K, E, s)$ satisfy Conjecture 1.1.

If we move, however, to 2-tangential bodies then we loose, in general, the negativity property of the real parts of the roots.

Theorem 1.2. There exists a 2 -tangential body $K \in \mathcal{K}^{15}$ such that $f\left(K, B_{15}, s\right)$ has a root with positive real part.

We remark that in a recent paper V. Katsnelson [8, Theorem 2.7/1.] also found independently another family of convex bodies for which the Steiner polynomial has roots with positive reals parts. The bodies are here certain flattered ellipsoids in high dimensions. In [8] not only the roots of Steiner polynomials are studied but also the roots of so called Weyl polynomials associated to a manifold. 
In the case of Steiner polynomials, the minimum dimension of a convex body $K$ such that $f(K, E, s)$ violates the negativity property of the roots is at least 6 . Based on the well-known inequalities

$$
\mathrm{W}_{i}(K ; E)^{2}-\mathrm{W}_{i-1}(K ; E) \mathrm{W}_{i+1}(K ; E) \geq 0, \quad 1 \leq i \leq n-1,
$$

which are particular cases of the Aleksandrov-Fenchel inequality (see e.g. [13, s. 6.3]), and on the Routh-Hurwitz criterion (see e.g. [9, p. 181]) one can check that $f(K, E, s)$ is a Hurwitz polynomial for $n \leq 5$, i.e., all its roots lie in the left half plane (see also [15, p. 103]).

In Section 4 we construct a 3-dimensional convex body violating the conjectured circumradius bound, more precisely,

Theorem 1.3. There exists $K \in \mathcal{K}_{0}^{3}$ such that all the real parts of the roots of $f\left(K, B_{3}, s\right)$ are greater than $-\mathrm{R}\left(K ; B_{3}\right)$.

Since $\mathrm{W}_{i}(K ; E)=\mathrm{W}_{n-i}(E ; K)$ we have $f(K, E, s)=s^{n} f(E, K, 1 / s)$. Hence, and on account of (1.2) it is not surprising that the body $K$ of the theorem above leads also to a counterexample for the inradius bound.

Corollary 1.1. There exists $K \in \mathcal{K}_{0}^{3}$ such that all the real parts of the roots of $f\left(B_{3}, K, s\right)$ are less than $-\mathrm{r}\left(B_{3} ; K\right)$.

Before giving the proofs of the theorems above we study in Section 2 the size of the roots of the relative Steiner polynomial $f(K, E, s)$. We prove upper and lower bounds for them in terms of the circumradius and the inradius.

\section{Bounds for the roots of the Steiner polynomial}

In the following we will use the inequalities

$$
\mathrm{r}(K ; E) \mathrm{W}_{i+1}(K ; E) \leq \mathrm{W}_{i}(K ; E) \leq \mathrm{R}(K ; E) \mathrm{W}_{i+1}(K ; E),
$$

for $i \in\{0, \ldots, n-1\}$. Since, up to translations, $\mathrm{r}(K ; E) E \subseteq K$ and $K \subseteq$ $\mathrm{R}(K ; E) E$ these inequalities are a direct consequence of the monotonicity of the mixed volumes (cf. e.g. [13, p. 277]).

Proposition 2.1. Let $K \in \mathcal{K}^{n}, E \in \mathcal{K}_{0}^{n}$, and let $\gamma_{i}, i=1, \ldots, n$, be the roots of the Steiner polynomial $f(K, E, s)$.

i) If $\operatorname{dim} K=m, m \geq 1$, the non-zero roots $\gamma_{i}$ are bounded by

$$
\frac{n-m+1}{m} \frac{\mathrm{W}_{n-m}(K ; E)}{\mathrm{W}_{n-m+1}(K ; E)} \leq\left|\gamma_{i}\right| \leq n \frac{\mathrm{W}_{n-1}(K ; E)}{\mathrm{W}_{n}(K ; E)} .
$$

The upper bound is best possible. In particular, we have $\mathrm{r}(K ; E) / n \leq$ $\left|\gamma_{i}\right| \leq n \mathrm{R}(K ; E)$. 
ii) $\left|\operatorname{Re}\left(\gamma_{1}\right)\right|+\cdots+\left|\operatorname{Re}\left(\gamma_{n}\right)\right| \geq n \mathrm{r}(K ; E)$.

iii) If $\operatorname{Re}\left(\gamma_{i}\right) \leq 0$ for all $i=1, \ldots, n$, then $\left|\operatorname{Re}\left(\gamma_{1}\right)\right|+\cdots+\left|\operatorname{Re}\left(\gamma_{n}\right)\right| \leq$ $n \mathrm{R}(K ; E)$.

Proof. Since $\operatorname{dim} K=m$ we have $\mathrm{W}_{i}(K ; E)=0$ for $i=0, \ldots, n-m-1$ and $\mathrm{W}_{i}(K ; E)>0$ for $i=n-m, \ldots, n$ (see [13, p. 277]). Hence the non-zero roots of the Steiner polynomial of $K$ are the roots of the polynomial on the right hand side in

$$
\frac{1}{s^{n-m}} f(K, E, s)=\sum_{i=0}^{m}\left(\begin{array}{c}
n \\
n-m+i
\end{array}\right) \mathrm{W}_{n-m+i}(K ; E) s^{i} .
$$

It is known that the roots of a polynomial $f(s)=a_{0}+a_{1} s+\cdots+a_{m} s^{m}$ with positive real coefficients $a_{j}$ lie in the ring $\rho_{1} \leq|s| \leq \rho_{2}$, where $\rho_{1}=$ $\min \left\{a_{j} / a_{j+1}\right\}$ and $\rho_{2}=\max \left\{a_{j} / a_{j+1}\right\}$, for $j=0,1, \ldots, m-1$, see e.g. $[9$, p. 137]. Hence in order to find the non-zero roots of $f(K, E, s)$ we have just to find the minimum and maximum of $\left(\begin{array}{c}n \\ j\end{array}\right) \mathrm{W}_{j}(K ; E) /\left(\left(\begin{array}{c}n \\ j+1\end{array}\right) \mathrm{W}_{j+1}(K ; E)\right)$, $j=n-m, \ldots, n-1$. By $(1.5)$ we see that $\mathrm{W}_{j}(K ; E) / \mathrm{W}_{j+1}(K ; E)$ is increasing in $j$, and since $\left(\begin{array}{c}n \\ j\end{array}\right) /\left(\begin{array}{c}n \\ j+1\end{array}\right)$ is also increasing we get

$$
\frac{n-m+1}{m} \frac{\mathrm{W}_{n-m}(K ; E)}{\mathrm{W}_{n-m+1}(K ; E)} \leq \frac{\left(\begin{array}{c}
n \\
j
\end{array}\right) \mathrm{W}_{j}(K ; E)}{\left(\begin{array}{c}
n \\
j+1
\end{array}\right) \mathrm{W}_{j+1}(K ; E)} \leq n \frac{\mathrm{W}_{n-1}(K ; E)}{\mathrm{W}_{n}(K ; E)},
$$

for $j=n-m, \ldots, n-1$, which shows the inequalities in i). The bounds in i) in terms of the inradius and the circumradius follow immediately with (2.1). Notice that if $m<n$ then $\mathrm{r}(K ; E)=0$. Hence the only non-trivial lower bound is obtained when $m=n$.

By i) we see that the only non-zero root of the Steiner polynomial of a line segment $K$, i.e., $\operatorname{dim} K=1$, is given by $\gamma=-n \mathrm{~W}_{n-1}(K ; E) / \mathrm{W}_{n}(K ; E)$. This shows that the upper bound in i) is best possible for any choice of the body $E \in \mathcal{K}_{0}^{n}$.

Since $f(K, E, s)=\mathrm{W}_{n}(K ; E) \prod_{i=1}^{n}\left(s-\gamma_{i}\right)$ we find that

$$
\gamma_{1}+\cdots+\gamma_{n}=-n \frac{\mathrm{W}_{n-1}(K ; E)}{\mathrm{W}_{n}(K ; E)} .
$$

Together with (2.1) we get

$n \mathrm{r}(K ; E) \leq\left|\gamma_{1}+\cdots+\gamma_{n}\right|=\left|\operatorname{Re}\left(\gamma_{1}\right)+\cdots+\operatorname{Re}\left(\gamma_{n}\right)\right| \leq\left|\operatorname{Re}\left(\gamma_{1}\right)\right|+\cdots+\left|\operatorname{Re}\left(\gamma_{n}\right)\right|$,

which shows ii). Finally, if all the roots have negative real part (as conjectured), we even have $\left|\operatorname{Re}\left(\gamma_{1}\right)\right|+\cdots+\left|\operatorname{Re}\left(\gamma_{n}\right)\right|=\left|\operatorname{Re}\left(\gamma_{1}\right)+\cdots+\operatorname{Re}\left(\gamma_{n}\right)\right|$ and as above we get by (2.1) the upper bound stated in iii). 


\section{The Steiner polynomial and tangential bodies}

A convex body $K \in \mathcal{K}^{n}$ containing the convex body $E \in \mathcal{K}^{n}$ is called a $p$ tangential body of $E, p \in\{0, \ldots, n-1\}$, if each support plane of $K$ that is not a support plane of $E$ contains only $(p-1)$-singular points of $K$ [13, p. 76]. Here a boundary point $x$ of $K$ is said to be an $r$-singular point of $K$ if the dimension of the normal cone in $x$ is at least $n-r$. For further characterizations and properties of $p$-tangential bodies we refer to [13, Section 2.2].

So a 0 -tangential body of $E$ is just the body $E$ itself and each $p$-tangential body of $E$ is also a $q$-tangential body for $p<q \leq n-1$. A 1-tangential body is usually called cap-body, and it can be seen as the convex hull of $E$ and countably many points such that the line segment joining any pair of those points intersects $E$.

If $K$ is a $p$-tangential body of $E$ then $\mathrm{r}(K ; E)=1$, and the following theorem gives a characterization of $n$-dimensional $p$-tangential bodies in terms of the quermassintegrals (cf. (2.1)).

Theorem 3.1 (Favard [5], [13, p. 367]). Let $K, E \in \mathcal{K}_{0}^{n}, K \subset E$, and let $p \in\{0, \ldots, n-1\}$. Then $\mathrm{W}_{0}(K ; E)=\mathrm{W}_{1}(K ; E)=\cdots=\mathrm{W}_{n-p}(K ; E)$ if and only if $K$ is a p-tangential body of $E$.

The next proposition shows that $n$-dimensional $p$-tangential bodies always satisfy the inradius bound of Conjecture 1.1.

Proposition 3.1. Let $p \in\{0, \ldots, n-1\}$, and let $K \in \mathcal{K}_{0}^{n}$ be a $p$-tangential body of $E \in \mathcal{K}_{0}^{n}$. Then there exists a root $\gamma$ of $f(K, E, s)$ such that $\operatorname{Re}(\gamma) \geq$ $-\mathrm{r}(K ; E)$.

Proof. Let $\gamma_{i}$, for $1 \leq i \leq n$, be the roots of $f(K, E, s)$. From $f(K, E, s)=$ $\mathrm{W}_{n}(K ; E) \prod_{i=1}^{n}\left(s-\gamma_{i}\right)$ we get

$$
\begin{aligned}
(-1)^{n} \mathrm{~W}_{0}(K ; E) & =\mathrm{W}_{n}(K ; E) \gamma_{1} \cdot \ldots \cdot \gamma_{n} \text { and } \\
(-1)^{n-1} n \mathrm{~W}_{1}(K ; E) & =\mathrm{W}_{n}(K ; E) \sum_{i=1}^{n} \prod_{j \neq i} \gamma_{j} .
\end{aligned}
$$

By Theorem 3.1 we have $\mathrm{W}_{0}(K ; E)=\mathrm{W}_{1}(K ; E)$ for any $p$-tangential body, and thus

$$
-n=-n \frac{\mathrm{W}_{1}(K ; E)}{\mathrm{W}_{0}(K ; E)}=\frac{1}{\gamma_{1}}+\cdots+\frac{1}{\gamma_{n}}=\operatorname{Re}\left(\frac{1}{\gamma_{1}}\right)+\cdots+\operatorname{Re}\left(\frac{1}{\gamma_{n}}\right) .
$$

Therefore, there exists a root $\gamma_{j}$, say, such that $\operatorname{Re}\left(1 / \gamma_{j}\right) \leq-1$, and so $\operatorname{Re}\left(\gamma_{j}\right) \geq-1=-\mathrm{r}(K ; E)$. 
Remark 3.1. Let $\gamma_{j}$ with $\operatorname{Re}\left(1 / \gamma_{j}\right) \leq-1$ be the root of the above proof of the polynomial $f(K, E, s)$. Since $\mathrm{W}_{i}(K ; E)=\mathrm{W}_{n-i}(E ; K)$ and thus $f(K, E, s)=s^{n} f(E, K, 1 / s)$ we see that $1 / \gamma_{j}$ is a root of $f(E, K, s) . B y(1.2)$ we have $\mathrm{R}(E ; K)=1$ and so we get that the polynomial $f(E, K, s)$ satisfies the circumradius bound.

Now we come to the proof of Theorem 1.1 and show that 1-tangential bodies (cap-bodies) fulfill the conjecture. We remark that the analogous 3-dimensional result for $E=B_{3}$ was already mentioned in [12, p. 65].

Proof of Theorem 1.1. If $K \in \mathcal{K}_{0}^{n}$ is a cap-body of $E \in \mathcal{K}_{0}^{n}$, Theorem 3.1 asserts that $\mathrm{W}_{0}(K ; E)=\mathrm{W}_{i}(K ; E)$, for all $i=1, \ldots, n-1$, and so we can rewrite the Steiner polynomial in the following way

$$
\begin{aligned}
f(K, E, s) & =\mathrm{W}_{0}(K ; E)\left[\sum_{i=0}^{n-1}\left(\begin{array}{c}
n \\
i
\end{array}\right) s^{i}+\frac{\mathrm{W}_{n}(K ; E)}{\mathrm{W}_{0}(K ; E)} s^{n}\right] \\
& =\mathrm{W}_{0}(K ; E)\left[(1+s)^{n}-(1-\alpha(K, E)) s^{n}\right],
\end{aligned}
$$

where $\alpha(K, E)=\mathrm{W}_{n}(K ; E) / \mathrm{W}_{0}(K ; E)$. Observe that $0<\alpha(K, E) \leq 1$ since $E \subseteq K$. So in this case all the roots $\gamma_{k}$ of $f(K, E, s)$ have to satisfy the equation $(1 / s+1)^{n}=1-\alpha(K, E)$ and so we obtain

$$
\frac{1}{\gamma_{k}}=-1+\sqrt[n]{1-\alpha(K, E)} \mathrm{e}^{\frac{2 \pi k}{n} \mathrm{i}}
$$

for $k=0, \ldots, n-1$. Hence all the real parts of $1 / \gamma_{k}$, and thus of $\gamma_{k}$ are non-positive which shows the negativity property of the roots stated in Conjecture 1.1. The inradius bound of the conjecture is guaranteed by Proposition 3.1 and it remains to verify the circumradius bound. The smallest real part among the roots $\gamma_{k}$ corresponds to $k=0$, i.e., it is

$$
\gamma_{0}=\frac{-1}{1-\sqrt[n]{1-\alpha(K, E)}}
$$

and we have to show that

$$
\frac{-1}{1-\sqrt[n]{1-\alpha(K, E)}} \leq-\mathrm{R}(K ; E),
$$

which is equivalent to

$$
1-\frac{\mathrm{W}_{n}(K ; E)}{\mathrm{W}_{0}(K ; E)} \geq\left(1-\frac{1}{\mathrm{R}(K ; E)}\right)^{n}
$$


In order to prove (3.1) we use the following inequality

$$
\left(\frac{\mathrm{W}_{n-1}(K ; E)}{\mathrm{W}_{0}(K ; E)}\right)^{\frac{n}{n-1}}-\frac{\mathrm{W}_{n}(K ; E)}{\mathrm{W}_{0}(K ; E)} \geq\left[\left(\frac{\mathrm{W}_{n-1}(K ; E)}{\mathrm{W}_{0}(K ; E)}\right)^{\frac{1}{n-1}}-\frac{1}{\mathrm{R}(K ; E)}\right]^{n}
$$

obtained in [11, Corollary 22]. Since $\mathrm{W}_{n-1}(K ; E)=\mathrm{W}_{0}(K ; E)$ we get immediately (3.1).

In contrast to 1-tangential bodies, the roots of Steiner polynomials of 2-tangential bodies do not fulfill all the properties of Conjecture 1.1, more precisely, some of their roots can have a positive real part if the dimension is large enough.

Proof of Theorem 1.2. On account of Theorem 3.1 we may write the Steiner polynomial $f\left(K, B_{n}, s\right)$ of a 2 -tangential body $K \in \mathcal{K}_{0}^{n}$ of $B_{n}$ as

$$
f\left(K, B_{n}, s\right)=\mathrm{W}_{0}(K)\left[\sum_{i=0}^{n-2}\left(\begin{array}{c}
n \\
i
\end{array}\right) s^{i}+n \frac{\mathrm{W}_{n-1}(K)}{\mathrm{W}_{0}(K)} s^{n-1}+\frac{\mathrm{W}_{n}(K)}{\mathrm{W}_{0}(K)} s^{n}\right] .
$$

We write $\beta(K)=\mathrm{W}_{n-1}(K) / \mathrm{W}_{0}(K)$ and $\alpha(K)=\mathrm{W}_{n}(K) / \mathrm{W}_{0}(K)$. All the roots of such a polynomial are non-zero. Since we are only interested in the negativity property of its roots we may divide by $s^{n}$ and with $\mu=1 / s$ it suffices to consider the polynomial

$$
h(K, \mu)=\sum_{i=2}^{n}\left(\begin{array}{c}
n \\
i
\end{array}\right) \mu^{i}+n \beta(K) \mu+\alpha(K) .
$$

Now it can be checked with a computer or by applying the Routh-Hurwitz criterion that the polynomial $\sum_{i=2}^{n}\left(\begin{array}{c}n \\ i\end{array}\right) \mu^{i}$ has a root with positive real part for $n=15$. Hence, if we find a 2 -tangential body $K \in \mathcal{K}_{0}^{15}$ for which $\beta(K)$ and $\alpha(K)$ can be arbitrarily small we get a counterexample to the negativity property of Conjecture 1.1. Observe that the roots of a polynomial are continuous functions of the coefficients of the polynomial (cf. e.g. [9, p. 3]).

In order to construct such a body let $P_{\lambda} \in \mathcal{K}_{0}^{3}, \lambda \geq 2$, be the pyramid over a square basis with vertices

$$
( \pm \lambda, \pm \lambda,-1)^{\top}, \quad\left(0,0,1+2 /\left(\lambda^{2}-1\right)\right)^{\top}
$$

The coordinates are chosen such that the largest ball contained in $P_{\lambda}$ is $B_{3}$ and that all 2-faces (facets) of $P_{\lambda}$ touch $B_{3}$. Next we embed $P_{\lambda}$ in the canonical way into $\mathbb{R}^{15}$ and consider $K_{\lambda}=\operatorname{conv}\left\{P_{\lambda}, B_{15}\right\} \in \mathcal{K}_{0}^{15}$. If $H$ is a support plane of $K_{\lambda}$ which is not a support plane of $B_{15}$ it must be a support plane 
of $P_{\lambda}$ and it can not contain any of the 2-faces of $P_{\lambda}$. Thus $H$ contains only 1-singular points of $K_{\lambda}$ and this shows that $K_{\lambda}$ is a 2-tangential body of $B_{15}$.

It is easy to see that for the pyramid $P_{\lambda}$ there exists a constant $c$ such that its 3-dimensional volume is not smaller than $c \lambda^{2}$. Hence there exists a constant $c_{n}$ depending only on the dimension such that $\mathrm{V}\left(K_{\lambda}\right)=\mathrm{W}_{0}\left(K_{\lambda}\right) \geq$ $c_{n} \lambda^{2}$. On the other hand, the circumradius of $K_{\lambda}$ is certainly less than $2 \lambda$ and so by (2.1) we have the bound

$$
\mathrm{W}_{n-1}\left(K_{\lambda}\right) \leq 2 \lambda \mathrm{V}\left(B_{15}\right)=2 \lambda \mathrm{W}_{n}\left(K_{\lambda}\right) .
$$

Thus

$$
\lim _{\lambda \rightarrow \infty} \beta\left(K_{\lambda}\right)=\lim _{\lambda \rightarrow \infty} \alpha\left(K_{\lambda}\right)=0,
$$

which shows that $h\left(K_{\lambda}, \mu\right)$ and thus $f\left(K_{\lambda}, B_{15}, s\right)$ has a root with positive real part if $\lambda$ is large enough.

Remark 3.2. It can be also checked that the polynomial $\sum_{i=3}^{n}\left(\begin{array}{c}n \\ i\end{array}\right) \mu^{i}$ has a root with positive real part in dimension $n=12$. Hence by an analogous argument and construction as above one can show that there exists a 3-tangential body $K \in \mathcal{K}_{0}^{12}$ of $B_{12}$ violating the negativity property of Conjecture 1.1.

Since the construction of this 12-dimensional counterexample is a bit more involved we omit it here. To close the section we give a 20-dimensional numerical counterexample based on an almost regular crosspolytope.

For $0<\lambda_{1} \leq \cdots \leq \lambda_{n}$ we denote by $C_{n}^{*}\left(\lambda_{1}, \ldots, \lambda_{n}\right)$ the orthogonal crosspolytope given by $C_{n}^{*}\left(\lambda_{1}, \ldots, \lambda_{n}\right)=\operatorname{conv}\left\{ \pm \lambda_{i} e_{i}: i=1, \ldots, n\right\}$, where $e_{i}$ denotes the $i$-th canonical unit vector. Analogously to the proof of Lemma 2.1 in [1] it can be shown:

Lemma 3.1. Let $F^{i}\left(\lambda_{l_{1}}, \ldots, \lambda_{l_{i+1}}\right)=\operatorname{conv}\left\{\lambda_{l_{1}} e_{l_{1}}, \ldots, \lambda_{l_{i+1}} e_{l_{i+1}}\right\}, 0 \leq i \leq n$, be an $i$-dimensional face of $C_{n}^{*}\left(\lambda_{1}, \ldots, \lambda_{n}\right), 1 \leq l_{1}<\cdots<l_{i+1} \leq n$. The external angle of the face $F^{i}\left(\lambda_{l_{1}}, \ldots, \lambda_{l_{i+1}}\right)$ is given by

$$
\frac{2^{n-i-1}}{\pi^{(n-i) / 2}} \frac{\sqrt{\sum_{k=1}^{i+1} \prod_{j \neq k} \lambda_{l_{j}}^{2}}}{\prod_{j=1}^{n} \lambda_{j}} \int_{0}^{\infty} \mathrm{e}^{-\left(\sum_{k=1}^{i+1} \frac{1}{\lambda_{l_{k}}^{2}}\right) x^{2}}\left(\prod_{j \neq\left\{l_{1}, \ldots, l_{i+1}\right\}} \int_{0}^{x} \mathrm{e}^{-\frac{y^{2}}{\lambda_{j}^{2}}} d y\right) d x .
$$

The $i$-face $F^{i}\left(\lambda_{l_{1}}, \ldots, \lambda_{l_{i+1}}\right)$ is the $i$-simplex $\operatorname{conv}\left\{\lambda_{l_{1}} e_{l_{1}}, \ldots, \lambda_{l_{i+1}} e_{l_{i+1}}\right\}$, and its $i$-th volume is given by

$$
\frac{1}{i !} \sqrt{\sum_{k=1}^{i+1} \prod_{j \neq k} \lambda_{l_{j}}^{2}}
$$

Since $C_{n}^{*}\left(\lambda_{1}, \ldots, \lambda_{n}\right)$ has $2^{i+1}$ equal $i$-faces of the type $F^{i}\left(\lambda_{l_{1}}, \ldots, \lambda_{l_{i+1}}\right)$ we get the following formulae for the quermassintegrals. 
Theorem 3.2. Let $0<\lambda_{1} \leq \cdots \leq \lambda_{n}$. The quermassintegrals of the orthogonal crosspolytope $C_{n}^{*}\left(\lambda_{1}, \ldots, \lambda_{n}\right)$ are given by:

$$
\begin{aligned}
\mathrm{W}_{0}\left(C_{n}^{*}\left(\lambda_{1}, \ldots, \lambda_{n}\right)\right) & =\frac{2^{n}}{n !} \lambda_{1} \cdot \ldots \cdot \lambda_{n}, \\
\mathrm{~W}_{1}\left(C_{n}^{*}\left(\lambda_{1}, \ldots, \lambda_{n}\right)\right) & =\frac{2^{n}}{n !} \sqrt{\sum_{i=1}^{n} \prod_{j \neq i} \lambda_{j}^{2}},
\end{aligned}
$$

and for $0 \leq i \leq n-2$,

$$
\begin{aligned}
\mathrm{W}_{n-i}\left(C_{n}^{*}\left(\lambda_{1}, \ldots, \lambda_{n}\right)\right)= & \frac{\kappa_{n-i}}{\left(\begin{array}{c}
n \\
i
\end{array}\right)} \frac{2^{n}}{i ! \pi^{(n-i) / 2}} \sum_{1 \leq l_{1}<\cdots<l_{i+1} \leq n}\left[\frac{\sum_{k=1}^{i+1} \prod_{j \neq k} \lambda_{l_{j}}^{2}}{\prod_{j=1}^{n} \lambda_{j}} \times\right. \\
& \left.\times \int_{0}^{\infty} \mathrm{e}^{-\left(\sum_{k=1}^{i+1} \frac{1}{\lambda_{l_{k}}^{2}}\right) x^{2}}\left(\prod_{j \neq\left\{l_{1}, \ldots, l_{i+1}\right\}} \int_{0}^{x} \mathrm{e}^{-\frac{y^{2}}{\lambda_{j}^{2}}} d y\right) d x\right] .
\end{aligned}
$$

Numerical computations show that the Steiner polynomial of the crosspolytope $C_{n}^{*}(\lambda, \stackrel{(n / 2)}{.}, \lambda, 1, \stackrel{(n / 2)}{.}, 1), n$ even, has roots with positive real parts for different values of $\lambda$ and $n$, e.g., for $n=20$ and $\lambda=0.01$.

\section{The in- and circumradius bound}

Now we deal with the part of Conjecture 1.1 regarding the inradius and the circumradius bounds and we are going to show that the conjectured properties do not hold in dimension 3. To this end we consider first planar convex bodies in $\mathbb{R}^{3}$ and show that the circumradius bound is false in general for that class of bodies. From such a planar body we can easily construct a 3-dimensional counterexample which also fails the inradius bound. The inradius and circumradius of a convex body with respect to the unit ball will be denoted by $\mathrm{r}(K)$ and $\mathrm{R}(K)$. For a planar convex body we denote, as usual, by $\mathrm{A}(K)$ and $\mathrm{p}(K)$ its area and perimeter, respectively. With this notation (1.4) can be rewritten in the case $E=B_{2}$ as

$$
\mathrm{A}(K)+\mathrm{p}(K) \rho+\pi \rho^{2} \leq 0, \text { if }-\mathrm{R}(K) \leq \rho \leq-\mathrm{r}(K) .
$$

The next lemma gives a characterization of those planar convex bodies in $\mathbb{R}^{3}$ failing the circumradius bound.

Lemma 4.1. Let $K \in \mathcal{K}^{3}$ be a planar convex body. All the roots of its Steiner polynomial $f\left(K, B_{3}, s\right)$ have real part greater than $-\mathrm{R}(K)$ if and only if $\mathrm{p}(K)^{2}<(128 /(3 \pi)) \mathrm{A}(K)$ and $\mathrm{p}(K)<(16 / 3) \mathrm{R}(K)$. 
Proof. Since $K \in \mathcal{K}^{3}$ is a planar body we have $\mathrm{W}_{0}(K)=0,3 \mathrm{~W}_{1}(K)=$ $2 \mathrm{~A}(K)$ and $3 \mathrm{~W}_{2}(K)=(\pi / 2) \mathrm{p}(K)$. Hence

$$
f\left(K, B_{3}, s\right)=s\left(2 \mathrm{~A}(K)+\frac{\pi}{2} \mathrm{p}(K) s+\frac{4}{3} \pi s^{2}\right) .
$$

The non-zero roots $\gamma_{1,2}$ are given by

$$
\gamma_{1,2}=3 \frac{-\mathrm{p}(K) \pm \sqrt{\mathrm{p}(K)^{2}-\frac{128}{3 \pi} \mathrm{A}(K)}}{16} .
$$

If $\mathrm{p}(K)^{2}-(128 /(3 \pi)) \mathrm{A}(K) \geq 0$, i.e., if $f\left(K, B_{3}, s\right)$ has only real roots, then we have

$$
3 \frac{-\mathrm{p}(K)-\sqrt{\mathrm{p}(K)^{2}-\frac{128}{3 \pi} \mathrm{A}(K)}}{16} \leq \frac{-\mathrm{p}(K)-\sqrt{\mathrm{p}(K)^{2}-4 \pi \mathrm{A}(K)}}{2 \pi} \leq-\mathrm{R}(K),
$$

where the last inequality follows from (4.1). Hence all the roots of the Steiner polynomial of $K$ have real parts greater than $-\mathrm{R}(K)$ if and only if $\mathrm{p}(K)^{2}-(128 /(3 \pi)) \mathrm{A}(K)<0$ and $-(3 / 16) \mathrm{p}(K)>-\mathrm{R}(K)$.

In order to find a convex body satisfying the conditions of the previous lemma we consider a symmetric lens $L$ with circumradius $\mathrm{R}(L)=1$ and perimeter $\mathrm{p}(L)=5.2<16 / 3$. It is well known (see e.g. [4, p. 88-89]) that symmetric lenses are the extremal sets of the inequality

$$
8 \phi \mathrm{A}(K) \leq \mathrm{p}(K)(\mathrm{p}(K)-4 \mathrm{R}(K) \cos \phi),
$$

where $\phi$ is determined as the positive solution of the equation $\mathrm{p}(K) \sin \phi=$ $4 \mathrm{R}(K) \phi$. In the case of a lens, $\phi$ is the half angle between the two circles of the lens, see Figure 1.

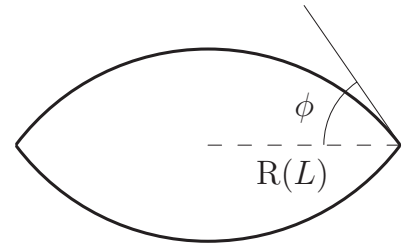

Figure 1: A symmetric lens $L$.

For our lens $L$ we obtain by the equality case in the above inequality $\mathrm{A}(L)=2.038627 \ldots$, which satisfies also the second inequality $\mathrm{p}(K)^{2}<$ $(128 /(3 \pi)) \mathrm{A}(K)$ of Lemma 4.1 . 
Hence $L$ is a 2 -dimensional convex body in $\mathbb{R}^{3}$ violating the circumradius bound. The roots of $f\left(L, B_{3}, s\right)$ are given by

$$
\gamma_{1,2}=-0.975 \pm 0.150823 \ldots \mathrm{i} \quad \text { and } \quad \gamma_{3}=0 \text {, }
$$

whose real parts are greater than $-\mathrm{R}(L)=-1$.

From such a set it is easy to obtain 3-dimensional convex bodies $K$ for which all the roots of $f\left(K, B_{3}, s\right)$ have real part greater than $-\mathrm{R}(K)$.

Proof of Theorem 1.3. Observe, in general, we have for $\nu, \rho \geq 0$ that $\mathrm{V}(K+\nu E+\rho E)=\mathrm{V}(K+(\nu+\rho) E)$ and thus

$$
f(K+\nu E, E, s)=\sum_{i=0}^{n}\left(\begin{array}{c}
n \\
i
\end{array}\right) \mathrm{W}_{i}(K ; E)(s+\nu)^{i}=f(K, E, s+\nu) .
$$

Hence the roots of $f(K+\nu E, E, s)$ are given by $\gamma_{i}-\nu$, where $\gamma_{i}$ are the roots of $f(K, E, s)$. Now let $L_{\nu}=L+\nu B_{3}$, where $L$ is the above 2-dimensional lens. Then $L_{\nu} \in \mathcal{K}_{0}^{3}$ for $\nu>0$ and the roots of $f\left(L_{\nu}, B_{3}, s\right)$ are $\gamma_{i}-\nu$, where $\gamma_{i}$ are given in (4.2). Thus all the real parts of $\gamma_{i}-\nu$ are greater than $-1-\nu=-\mathrm{R}\left(L_{\nu}, B_{3}\right)$.

Finally we deal with the inradius bound. To this end let $L_{\nu}$ be as in the proof above. Since $\mathrm{W}_{i}\left(L_{\nu} ; B_{3}\right)=\mathrm{W}_{3-i}\left(B_{3} ; L_{\nu}\right)$ we get

$$
\begin{aligned}
f\left(B_{3}, L_{\nu}, s\right)= & \left(2 \mathrm{~A}(L) \nu+\frac{\pi}{2} \mathrm{p}(L) \nu^{2}+\frac{4}{3} \pi \nu^{3}\right) s^{3} \\
& +\left(2 \mathrm{~A}(L)+\pi \mathrm{p}(L) \nu+4 \pi \nu^{2}\right) s^{2}+\left(\frac{\pi}{2} \mathrm{p}(L)+4 \pi \nu\right) s+\frac{4}{3} \pi .
\end{aligned}
$$

For instance, for $\nu=1$ it can be checked that the roots of this polynomial are

$$
\gamma_{1,2}=-0.503393 \ldots \pm 0.038442 \ldots \mathrm{i} \quad \text { and } \quad \gamma_{3}=-1,
$$

whose real parts are smaller than $-1 / 2=-1 / \mathrm{R}\left(L_{1}, B_{3}\right)=-\mathrm{r}\left(B_{3}, L_{1}\right)$.

This provides the required counterexample to the inradius bound of Conjecture 1.1 stated in Corollary 1.1.

We remark that the above construction in the proof of Theorem 1.3 and Corollary 1.1 works with any symmetric lens $L$ with $\mathrm{R}(L)=1$ and perimeter $\mathrm{p}(L) \in\left[\mathrm{p}_{0}, 16 / 3\right]$, where $\mathrm{p}_{0}=4 \phi_{0} / \sin \phi_{0}=5.052 \ldots$ and $\phi_{0}$ is the smallest positive solution of $\phi-\sin \phi \cos \phi=(3 \pi / 16) \phi^{2}$.

Acknowledgements. We would like to thank Vitali Milman and Rolf Schneider for bringing the paper of V. Katsnelson [8] to our attention. 


\section{References}

[1] Betke, U. And Henk, M.: Intrinsic volumes and lattice points of crosspolytopes. Monatsh. Math. 115 (1993), no. 1-2, 27-33.

[2] Blaschke, W.: Vorlesungen über Integralgeometrie. Deutscher Verlag der Wissenschaften, Berlin, 1995, 3rd ed.

[3] Bonnesen, T.: Les problèmes des isopérimètres et des isépiphanes. Collection de monographies sur la théorie des fonctions. Gauthier-Villars, Paris, 1929.

[4] Bonnesen, T. And Fenchel, W.: Theorie der konvexen Körper. Springer-Verlag, Berlin-New York, 1974. English translation: Theory of convex bodies. Edited by L. Boron, C. Christenson and B. Smith. BCS Associates, Moscow, ID, 1987.

[5] Favard, J.: Sur les corps convexes. J. Math. Pures Appl. (9) 12 (1933), 219-282.

[6] Flanders, H.: A proof of Minkowski's inequality for convex curves. Amer. Math. Monthly 75 (1968), 581-593.

[7] Hernández Cifre, M. A. and Saorín, E.: On the roots of the Steiner polynomial of a 3-dimensional convex body. Adv. Geom. 7 (2007), 275-294.

[8] Katsnelson, V.: On H. Weyl and H. Minkowski polynomials. Preprint, 2007. http://arxiv.org/abs/math/0702139.

[9] Marden, M.: Geometry of polynomials. Second edition. Mathematical Surveys 3. American Mathematical Society, Providence, R.I., 1966.

[10] OdA, T.: Convex bodies and algebraic geometry. An introduction to the theory of toric varieties. Results in Mathematics and Related Areas (3) 15. Springer-Verlag, Berlin, 1988.

[11] Sangwine-Yager, J. R.: Bonnesen-style inequalities for Minkowski relative geometry. Trans. Amer. Math. Soc. 307 (1988), no. 1, 373-382.

[12] Sangwine-Yager, J. R.: Mixed volumes. In: Handbook of convex geometry, Vol. A, B (P. M. Gruber and J. M. Wills eds.), 43-71. North-Holland, Amsterdam, 1993.

[13] Schneider, R.: Convex Bodies: The Brunn-Minkowski theory. Encyclopedia of Mathematics and its Applications 44. Cambridge University Press, Cambridge, 1993.

[14] Steiner, J.: Über parallele Flächen. Monatsber. Preuss. Akad. Wiss. (1840), 114-118. Also in: Ges. Werke, Vol II, 245-308. Reimer, Berlin, 1882.

[15] Teissier, B.: Bonnesen-type inequalities in algebraic geometry. I. Introduction to the problem. In Seminar on Differential Geometry, 85-105. Annals of Mathematical Studies 102. Princeton University Press, Princeton, N. J., 1982. 
[16] WiLLS, J. M.: Minkowski's successive minima and the zeros of a convexityfunction. Monatsh. Math. 109 (1990), 157-164.

Recibido: 26 de abril de 2007

Revisado: 30 de julio de 2007

Martin Henk

Institut für Algebra und Geometrie

Otto-von-Guericke Universität Magdeburg

Universitätsplatz 2

D-39106-Magdeburg, Germany

henk@math . uni-magdeburg. de

María A. Hernández Cifre

Departamento de Matemáticas

Universidad de Murcia

Campus de Espinardo

30100-Murcia, Spain

mhcifre@um.es

This work has been developed during a research stay of the second author at the Universität Magdeburg, Germany, supported by Programa Nacional de ayudas para la movilidad de profesores de universidad e investigadores españoles y extranjeros (MEC), Ref. PR2006-0351. Second author is supported in part by Dirección General de Investigación (MEC) MTM2004-04934-C04-02 and by Fundación Séneca (C.A.R.M.) $00625 / \mathrm{PI} / 04$. 